\title{
Current Status of Interpretation of Small Bowel Capsule Endoscopy
}

\author{
Su Hwan Kim 1 , Dong-Hoon Yang ${ }^{2}$ and Jin Su Kim ${ }^{3}$ \\ ${ }^{1}$ Department of Internal Medicine, Seoul Metropolitan Government Seoul National University Boramae Medical Center, Seoul National \\ University College of Medicine, Seoul, ${ }^{2}$ Department of Gastroenterology, Asan Medical Center, University of Ulsan College of Medicine, \\ Seoul, ${ }^{3}$ Division of Gastroenterology, Department of Internal Medicine, Seoul St. Mary's Hospital, The Catholic University of Korea College of \\ Medicine, Seoul, Korea
}

Capsule endoscopy (CE) has revolutionized direct small bowel imaging and is widely used in clinical practice. Remote visualization of bowel images enables painless, well-tolerated endoscopic examinations. Small bowel CE has a high diagnostic yield and the ability to examine the entire small bowel. The diagnostic yield of CE relies on lesion detection and interpretation. In this review, issues related to lesion detection and interpretation of $\mathrm{CE}$ have been addressed, and the current status of automated reading software development has been reviewed. Clinical significance of an external real-time image viewer has also been described. Clin Endosc 2018;51:329-333

Key Words: Capsule endoscopy; Small bowel; Interpretation; Software

\section{INTRODUCTION}

Capsule endoscopy (CE) was introduced in 2000 and has revolutionized direct small bowel imaging. ${ }^{1} \mathrm{CE}$ is now widely used in clinical practice, and more than 2,000,000 capsules have been swallowed worldwide. ${ }^{2}$ By separating the imaging device from the monitor screen, optical cabling used in conventional endoscopy is no longer necessary in CE. Remote visualization of bowel images in CE enabled painless, non-invasive, well-tolerated endoscopic examination, with no need for sedation. ${ }^{3}$

Received: June 3, 2018 Revised: July 14, 2018

Accepted: July 18, 2018

Correspondence: Dong-Hoon Yang and Jin Su Kim

Dong-Hoon Yang

Department of Gastroenterology, Asan Medical Center, University of Ulsan College of Medicine, 88 Olympic-ro 43-gil, Songpa-gu, Seoul 05505, Korea

Tel: +82-2-3010-5809, Fax: +82-2-3010-6517, E-mail: dhyang@amc.seoul.kr ORCID: https://orcid.org/0000-0001-7756-2704

Jin Su Kim

Division of Gastroenterology, Department of Internal Medicine, Seoul St. Mary's Hospital, The Catholic University of Korea College of Medicine, 222 Banpo-daero, Seocho-gu, Seoul 06591, Korea

Tel: +82-2-2258-2044, Fax: +82-2-2258-2089, E-mail: jinsu23@naver.com ORCID: https://orcid.org/0000-0002-3397-3189

(c) This is an Open Access article distributed under the terms of the Creative Commons Attribution Non-Commercial License (http://creativecommons.org/ licenses/by-nc/3.0) which permits unrestricted non-commercial use, distribution, and reproduction in any medium, provided the original work is properly cited.
As direct endoscopic observation is superior to barium studies, esophagogastroduodenoscopy and colonoscopy have almost replaced upper gastrointestinal series and barium enemas. Likewise, CE of the small bowel has made the small bowel series almost unnecessary. Small bowel CE has high diagnostic yield and the ability to examine the entire small bowel. The diagnostic yield of $\mathrm{CE}$ relies on lesion detection and interpretation. Lesion detection is affected by the quality of imaging and the number of images taken per second. ${ }^{4,5}$ Lesion interpretation takes considerable time and requires focused attention. There have been attempts to use computational software programs to reduce $\mathrm{CE}$ reading time and automatically detect abnormalities. Here, we briefly describe current status and perspectives on the interpretation of small bowel CE.

\section{LESION DETECTION IN SMALL BOWEL CAPSULE ENDOSCOPY}

Since the introduction of $\mathrm{CE}$ in clinical practice, there have been many technical developments. The picture quality of $\mathrm{CE}$ imaging has been improved by the introduction of newer devices with improved optical properties. The earliest M2A ${ }^{\circledR}$ capsule (Given Imaging Ltd., Yoqneam, Israel) had only a single element lens that exhibited peripheral image distortion. 
Newer PillCam ${ }^{\circledR}$ SB2 and SB3 capsules (Medtronic, Dublin, Ireland) have multi-element lenses, with distortion-free fields of view of $156^{\circ}$. In the older capsules, light intensity was not modulated according to the distance from the mucosa, which resulted in glare or dim lighting of images according to the distance of the capsule from the mucosa. Newer devices with adaptive illumination have control of automated illumination adjustment, which provide uniform lighting irrespective of the distance from the mucosa. ${ }^{6}$

Capsules move in the gastrointestinal tract with peristalsis, which is unpredictable. Unpredictable rapid peristalsis can result in misses or incomplete evaluation of the small bowel. ${ }^{7}$ The risk of missing lesions is high for lesions that are located in the duodenum or proximal jejunum, where the capsule moves more rapidly than in other parts of the small bowel. ${ }^{8}$ The PillCam ${ }^{\circledR}$ SB2 system could capture 2 frames per second. The newer generation PillCam ${ }^{\circledR}$ SB3 system has an adaptive frame rate of 2-6 frames per second. More pictures are taken when the capsule is moving more rapidly, and fewer pictures are taken when the device is moving slowly, which leads to a reduction of duplicated images. ${ }^{9} \mathrm{CE}$ with an imager at each end of the capsule detects more lesions, ${ }^{10}$ but increasing the number of images leads to an increase in reading time.

\section{INTERPRETATION OF SMALL BOWEL CAPSULE ENDOSCOPY}

Unlike conventional endoscopy, the CE procedure itself does not require special skills. ${ }^{11}$ Patients are required to just swallow a capsule. Thus, accurate diagnosis with CE depends on the reviewer's ability to read and interpret the images. The problem is that it takes more than an hour to read the $50,000-100,000$ images captured. In this passive form of endoscopy, reading 50,000-100,000 images of the same organ is monotonous and difficult. During CE reading, no additional stimulus is provided to manipulate the instrument, and generally no communication occurs with the patient or staff. Concentration and alertness are required for this time-consuming process of CE image reading. The key point is the reviewer's ability to observe without being distracted. ${ }^{3}$ Reports of missed lesions ${ }^{12,13}$ and significant interpersonal variability ${ }^{14,15}$ may be in part due to interpretation failure and reflect the difficulty in sustaining concentration.

The video can be run in a single, double, or quadruple view. The double or quadruple view has been recommended to improve reading efficiency and the lesion detection rate by interpreters. ${ }^{16}$ With an increase in the video speed, the risk of missing significant lesions may increase. ${ }^{3}$ A previous study suggested that $\mathrm{CE}$ recordings should be read at a maximum speed of 15 frames per second for an acceptable detection rate. $^{16}$

In a study, CE trainees with more than 20 interpretations got higher scores. Thus, it has been suggested that trainees need to perform at least 20 supervised capsule interpretations before they are certified. ${ }^{17}$ However, other studies have shown no difference in the detection rate among readers with different experience. $^{18}$

As significant time is required for $\mathrm{CE}$ interpretation, taking help of non-physicians for interpretation might be an option. There have been studies comparing CE interpretation between experts, non-experts, and nurses. For non-expert CE readers, some level of prior conventional endoscopy experience was necessary, but high-level experience did not add benefit. ${ }^{1719}$ Nurses showed no differences in recognizing significant lesions compared with physicians. ${ }^{20,21}$ Taking help of non-physicians for CE interpretation might improve efficiency and reduce the cost of interpretation. ${ }^{21}$ Qualified nurses and technicians can be accepted as prereaders of $\mathrm{CE}$, as the responsibility of making a diagnosis remains with the physician.

The primary goal of small bowel CE is to visualize the small bowel. However, careful observation of gastric and colonic images to find missed lesions is required in patients with obscure gastrointestinal bleeding. CE may detect $25 \%$ of upper gastrointestinal lesions in the stomach or esophagus that were not detected on conventional endoscopy previously. ${ }^{22,23}$

\section{READING SOFTWARE}

Another approach to reduce CE reading time is using special software programs to select images for subsequent $\mathrm{CE}$ reading. The first software designed for this goal was the suspected blood indicator (SBI, Medtronic), an automatic system that could pick up frames with red pixels (Fig. 1). The SBI system was developed to detect red lesions responsible for anemia or bleeding. However, even in patients with active bowel bleeding, the sensitivity of SBI was less than $60 \% .^{24}$ The accuracy of the SBI system proved suboptimal and can be used only as a supportive tool. ${ }^{25}$

The QuickView mode is an automated fast reading tool available on Rapid software (Medtronic), which scans all images and scores them according to the level of significance. The number of "frames of interest" images can be set as a percentage (e.g., 10\%, 20\%, 80\%) of the full images. Then, QuickView displays selected images according to the percentage level set by the user. ${ }^{26}$ The QuickView significantly reduced $\mathrm{CE}$ reading time but was associated with considerable diagnostic miss rates. ${ }^{27}$ The QuickView may be used to diagnose Crohn's disease of the small bowel despite a significant num- 
ber of missed lesions. However, viewing the terminal ileum in standard view was recommended to avoid false negative cases. $^{28}$ The QuickView can also be helpful in overt obscure gastrointestinal bleeding in an urgent inpatient setting. ${ }^{5}$

Automated detection of lesions has recently undergone intense study. Various computational systems have been proposed to improve the diagnostic ability of CE. Such systems can analyze CE images using algorithms that quantify image features discriminating between normal and abnormal. These systems consider image features such as color, texture, or shape. Image classification is generally based on machine learning algorithms. These algorithms are trainable with annotated images, including information on the location and pathology. ${ }^{29} \mathrm{~A}$ recent study showed that a deep learning system had high sensitivity and specificity for identifying diabetic retinopathy. ${ }^{30}$ Deep neural networks also showed dermatologist-level classification of skin cancer. ${ }^{31}$ However, integrating machine learning algorithms into $\mathrm{CE}$ interpretation is not easy because a considerable number of image annotations are required to develop image recognition software. ${ }^{29,32}$ Various studies have been reported on automated detection of abnormal findings in CE. Most studies investigated computer-based methods for the detection of blood, ulcers, and polyps. ${ }^{33-35}$ Some studies considered petechiae, hookworms, uninformative dark parts, or intestinal contents such as bubbles. ${ }^{36-38}$ Most studies focused on one or only a few abnormalities, and this is different from actual clinical situations in which discrimination among at least 10 abnormal findings is necessary. The diversity of lesions makes automatic lesion detection more challenging. One approach based on color saliency detected various abnormalities (polyp, ulcer, hemorrhage, angioectasia, chylous cyst, lymphangiectasia, stenosis, etc.) without exclud-

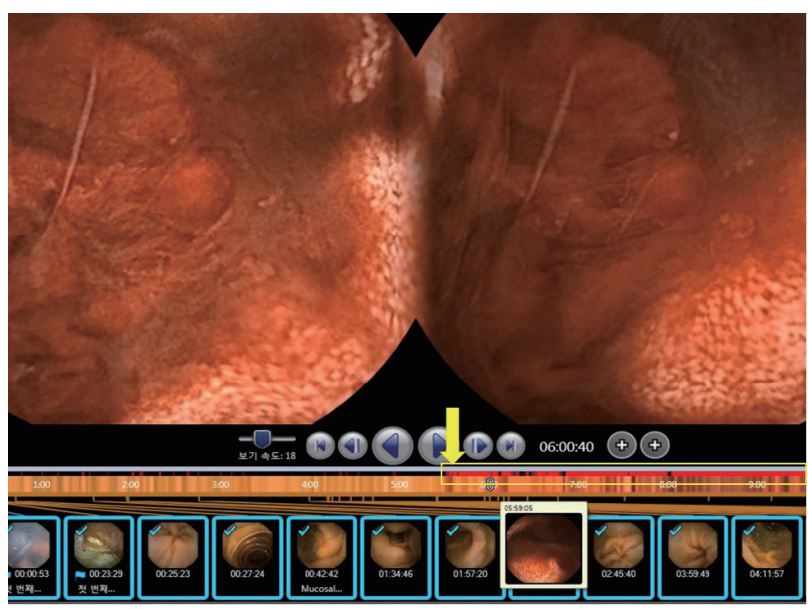

Fig. 1. A representative case with suspected blood indicator showed true blood in the small intestine. Suspected blood indicators are marked with red bands (in the yellow box indicated by a yellow arrow). ing intestinal content from the evaluation, and the average accuracy was $94.0 \%{ }^{32}$ Although technological breakthrough of automated reading software is being sought, conventional reading is still required and should not be replaced by reading software. ${ }^{39}$

\section{EXTERNAL REAL-TIME IMAGE VIEWER}

A recently introduced data recorder DR $3^{\circledR}$ (Medtronic) is equipped with a screen that shows real-time images during ongoing examination (Fig. 2). A real-time image viewer may shorten the duration of CE since the procedure may be terminated after the cecum is seen. ${ }^{7}$ In a case-control study, patients using a real-time image viewer coupled with prespecified actions showed higher completion rates and diagnostic yield. ${ }^{40}$ In patients with risk of delayed gastric emptying, the real-time image viewer may guide appropriate interventions to complete the examination. ${ }^{39}$

Evaluation of real-time CE imaging could change the management of gastrointestinal bleeding (Fig. 3). In a report, bedside real-time $\mathrm{CE}$ reading in an emergency department detected bleeding sites in one-third of the patients, saving time before therapeutic interventions. ${ }^{41}$

\section{LOCALIZATION OF CAPSULE AND LESION}

Capsules do not provide information regarding localization while passing through the gastrointestinal tract. ${ }^{42}$ The position and orientation of the capsule can be described with

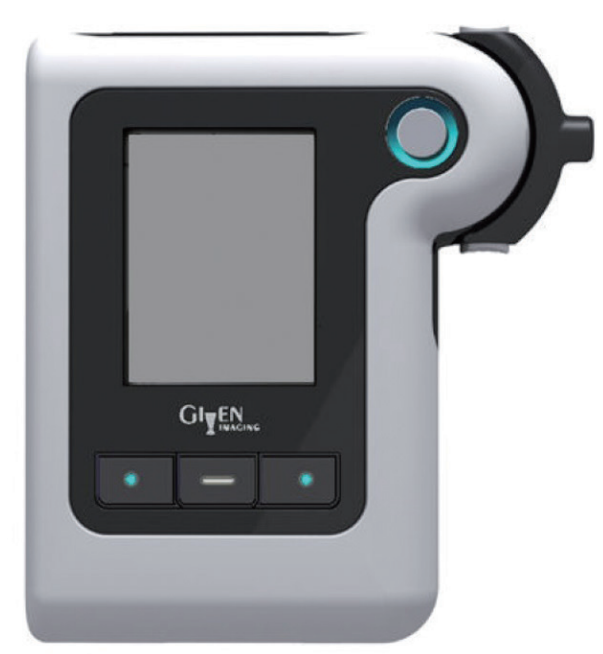

Fig. 2. External real-time image viewer. 


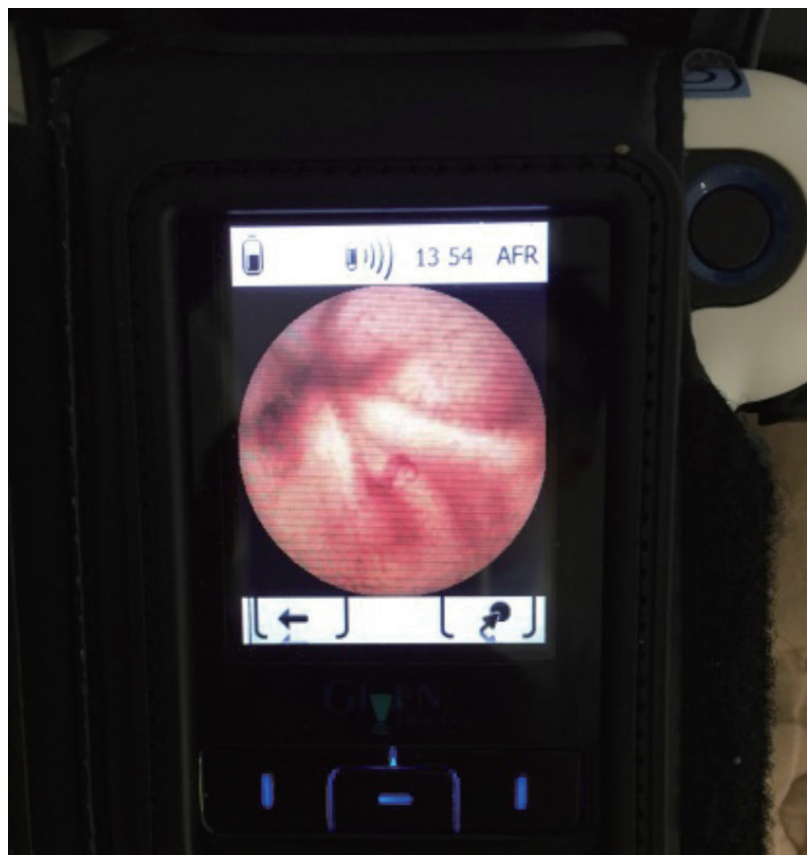

Fig. 3. External real-time image viewer indicating active small bowel bleeding.

regard to anatomical landmarks (diagnostic localization) or by a coordinate position in space (global localization). ${ }^{43} \mathrm{~A}$ study used radiofrequency triangulation around the abdominal wall and provided three-dimensional localization, which showed an average spatial error of $13.26 \mathrm{~cm}^{3}$ compared with plain radiography. ${ }^{44}$ However, because the gastrointestinal tract has a deformable nature, we cannot depend only on global localization to locate target areas. ${ }^{45}$ Localization of the capsule within the body is important for determining the enteroscopy route (oral or anal) or the planning of therapeutic interventions. ${ }^{46}$ The capsule does not move at a uniform speed through the gastrointestinal tract. Within current systems, approximate localization is based on transit time. Once the pylorus and cecum are identified, the location of lesions within the small bowel is an estimate based on the time from these two points. ${ }^{42}$ Small bowel transit time (SBTT) is calculated by subtracting the time of the first duodenal image from the first cecal image. If there is an abnormal finding during $\mathrm{CE}$, the percent of SBTT can guide the subsequent enteroscopy route for intervention. If the lesion is within the first $2 / 3$ of the SBTT, an antegrade approach for enteroscopy is needed. If the lesion is beyond the first $2 / 3$ of the SBTT, a retrograde approach is used.' The recently developed OdoCapsule provides real-time distance from duodenal entry to exit from the ileum via mounted passive wheels on its side that operate as an odometer. OdoCapsule has 3 extendable legs with rotating wheels, and stabilizes capsule transit by reducing side-to-side and tumbling movements. Ex vivo animal studies show that this model may act as an odometer, measuring distance by wheel rotation. The stability in capsule transit may aid in the development of computer image recognition systems. ${ }^{47}$

\section{CONCLUSIONS}

Since introduction into clinical practice, $\mathrm{CE}$ has proven its high diagnostic ability in various gastrointestinal diseases. The achievements of CE have exceeded what was previously expected. Novel CE technologies are being developed to improve diagnostic yield. Initially designed with the aim of evaluating the small bowel, $\mathrm{CE}$ is now expanding its fields of application to the entire gastrointestinal tract including esophagus, stomach, and colon. It may replace diagnostic conventional endoscopy of the entire gastrointestinal tract and perform therapeutic interventions in the future.

As of now, automated fast reading software cannot replace conventional reading. However, image recognition software is actively being investigated, and is expected to speed up the $\mathrm{CE}$ interpretation process. Given that artificial intelligence (AI)based image diagnosis is being actively investigated in various clinical fields, AI may provide a much-improved automated interpretation of $\mathrm{CE}$ in the near future.

\section{Conflicts of Interest}

The authors have no financial conflicts of interest.

\section{REFERENCES}

1. Iddan G, Meron G, Glukhovsky A, Swain P. Wireless capsule endoscopy. Nature 2000;405:417.

2. Eliakim R. Video capsule endoscopy of the small bowel. Curr Opin Gastroenterol 2013;29:133-139.

3. McAlindon ME, Ching HL, Yung D, Sidhu R, Koulaouzidis A. Capsule endoscopy of the small bowel. Ann Transl Med 2016;4:369.

4. Saurin JC, Beneche N, Chambon C, Pioche M. Challenges and future of wireless capsule endoscopy. Clin Endosc 2016;49:26-29.

5. Koulaouzidis A, Iakovidis DK, Karargyris A, Plevris JN. Optimizing lesion detection in small-bowel capsule endoscopy: from present problems to future solutions. Expert Rev Gastroenterol Hepatol 2015;9:217235.

6. Fisher LR, Hasler WL. New vision in video capsule endoscopy: current status and future directions. Nat Rev Gastroenterol Hepatol 2012;9:392405.

7. Goenka MK, Majumder S, Goenka U. Capsule endoscopy: present status and future expectation. World J Gastroenterol 2014;20:10024-10037.

8. Singeap AM, Stanciu C, Trifan A. Capsule endoscopy: the road ahead. World J Gastroenterol 2016;22:369-378.

9. Barkin JA, Barkin JS. Video capsule endoscopy: technology, reading, and troubleshooting. Gastrointest Endosc Clin N Am 2017;27:15-27.

10. Triantafyllou K, Papanikolaou IS, Papaxoinis K, Ladas SD. Two cameras detect more lesions in the small-bowel than one. World J Gastroenterol 2011;17:1462-1467. 
11. Drew K, McAlindon ME, Sanders DS, Sidhu R. The nurse endoscopist: moving ahead with time. Gastroenterol Nurs 2013;36:209-213.

12. Hakim FA, Alexander JA, Huprich JE, Grover M, Enders FT. CT-enterography may identify small bowel tumors not detected by capsule endoscopy: eight years experience at Mayo Clinic Rochester. Dig Dis Sci 2011;56:2914-2919.

13. Postgate A, Despott E, Burling D, et al. Significant small-bowel lesions detected by alternative diagnostic modalities after negative capsule endoscopy. Gastrointest Endosc 2008;68:1209-1214.

14. Zheng Y, Hawkins L, Wolff J, Goloubeva O, Goldberg E. Detection of lesions during capsule endoscopy: physician performance is disappointing. Am J Gastroenterol 2012;107:554-560.

15. Pezzoli A, Cannizzaro R, Pennazio M, et al. Interobserver agreement in describing video capsule endoscopy findings: a multicentre prospective study. Dig Liver Dis 2011;43:126-131.

16. Shim KN, Jeon SR, Jang HJ, et al. Quality indicators for small bowel capsule endoscopy. Clin Endosc 2017;50:148-160.

17. Rajan E, Iyer PG, Oxentenko AS, et al. Training in small-bowel capsule endoscopy: assessing and defining competency. Gastrointest Endosc 2013;78:617-622.

18. Rondonotti E, Soncini M, Girelli CM, et al. Can we improve the detection rate and interobserver agreement in capsule endoscopy? Dig Liver Dis 2012;44:1006-1011.

19. Sidhu R, Sakellariou P, McAlindon ME, et al. Is formal training necessary for capsule endoscopy? The largest gastroenterology trainee study with controls. Dig Liver Dis 2008;40:298-302.

20. Niv Y, Niv G. Capsule endoscopy examination--preliminary review by a nurse. Dig Dis Sci 2005;50:2121-2124.

21. Dokoutsidou H, Karagiannis S, Giannakoulopoulou E, et al. A study comparing an endoscopy nurse and an endoscopy physician in capsule endoscopy interpretation. Eur J Gastroenterol Hepatol 2011;23:166-170.

22. Lepileur L, Dray X, Antonietti M, et al. Factors associated with diagnosis of obscure gastrointestinal bleeding by video capsule enteroscopy. Clin Gastroenterol Hepatol 2012;10:1376-1380.

23. Tacheci I, Devière J, Kopacova M, Douda T, Bures J, Van Gossum A. The importance of upper gastrointestinal lesions detected with capsule endoscopy in patients with obscure digestive bleeding. Acta Gastroenterol Belg 2011;74:395-399.

24. Buscaglia JM, Giday SA, Kantsevoy SV, et al. Performance characteristics of the suspected blood indicator feature in capsule endoscopy according to indication for study. Clin Gastroenterol Hepatol 2008;6:298301.

25. D'Halluin PN, Delvaux M, Lapalus MG, et al. Does the "Suspected Blood Indicator" improve the detection of bleeding lesions by capsule endoscopy? Gastrointest Endosc 2005;61:243-249.

26. Saurin JC, Lapalus MG, Cholet F, et al. Can we shorten the small-bowel capsule reading time with the "Quick-view" image detection system? Dig Liver Dis 2012;44:477-481.

27. Westerhof J, Koornstra JJ, Weersma RK. Can we reduce capsule endoscopy reading times? Gastrointest Endosc 2009;69:497-502.

28. Halling ML, Nathan T, Kjeldsen J, Jensen MD. High sensitivity of quick view capsule endoscopy for detection of small bowel Crohn's disease. J Gastroenterol Hepatol 2014;29:992-996.

29. Iakovidis DK, Koulaouzidis A. Software for enhanced video capsule endoscopy: challenges for essential progress. Nat Rev Gastroenterol
Hepatol 2015;12:172-186.

30. Ting DSW, Cheung CY, Lim G, et al. Development and validation of a deep learning system for diabetic retinopathy and related eye diseases using retinal images from multiethnic populations with diabetes. JAMA 2017;318:2211-2223.

31. Esteva A, Kuprel B, Novoa RA, et al. Dermatologist-level classification of skin cancer with deep neural networks. Nature 2017;542:115-118.

32. Iakovidis DK, Koulaouzidis A. Automatic lesion detection in capsule endoscopy based on color saliency: closer to an essential adjunct for reviewing software. Gastrointest Endosc 2014;80:877-883.

33. Karargyris A, Bourbakis N. Detection of small bowel polyps and ulcers in wireless capsule endoscopy videos. IEEE Trans Biomed Eng 2011;58:2777-2786.

34. Li B, Meng MQ, Lau JY. Computer-aided small bowel tumor detection for capsule endoscopy. Artif Intell Med 2011;52:11-16.

35. Li BP, Meng MQ. Comparison of several texture features for tumor detection in CE images. J Med Syst 2012;36:2463-2469.

36. Wu X, Chen H, Gan T, Chen J, Ngo CW, Peng Q. Automatic hookworm detection in wireless capsule endoscopy images. IEEE Trans Med Imaging 2016;35:1741-1752.

37. Seguí S, Drozdzal M, Vilariño F, et al. Categorization and segmentation of intestinal content frames for wireless capsule endoscopy. IEEE Trans Inf Technol Biomed 2012;16:1341-1352.

38. Fan Y, Meng MQ, Li B. A novel method for informative frame selection in wireless capsule endoscopy video. Conf Proc IEEE Eng Med Biol Soc 2011;2011:4864-4867.

39. Rondonotti E, Spada C, Adler S, et al. Small-bowel capsule endoscopy and device-assisted enteroscopy for diagnosis and treatment of small-bowel disorders: European Society of Gastrointestinal Endoscopy (ESGE) technical review. Endoscopy 2018;50:423-446.

40. Shiotani A, Honda K, Kawakami M, et al. Use of an external real-time image viewer coupled with prespecified actions enhanced the complete examinations for capsule endoscopy. J Gastroenterol Hepatol 2011;26:1270-1274

41. Rubin M, Hussain SA, Shalomov A, Cortes RA, Smith MS, Kim SH. Live view video capsule endoscopy enables risk stratification of patients with acute upper GI bleeding in the emergency room: a pilot study. Dig Dis Sci 2011;56:786-791.

42. Fischer D, Schreiber R, Levi D, Eliakim R. Capsule endoscopy: the localization system. Gastrointest Endosc Clin N Am 2004;14:25-31.

43. Slawinski PR, Obstein KL, Valdastri P. Capsule endoscopy of the future: what's on the horizon? World J Gastroenterol 2015;21:10528-10541.

44. Marya N, Karellas A, Foley A, Roychowdhury A, Cave D. Computerized 3-dimensional localization of a video capsule in the abdominal cavity: validation by digital radiography. Gastrointest Endosc 2014;79:669674 .

45. Slawinski PR, Obstein KL, Valdastri P. Emerging issues and future developments in capsule endoscopy. Tech Gastrointest Endosc 2015;17:4046.

46. Li X, Chen H, Dai J, Gao Y, Ge Z. Predictive role of capsule endoscopy on the insertion route of double-balloon enteroscopy. Endoscopy 2009;41:762-766

47. Karargyris A, Koulaouzidis A. OdoCapsule: next-generation wireless capsule endoscopy with accurate lesion localization and video stabilization capabilities. IEEE Trans Biomed Eng 2015;62:352-360. 